ReVISIÓN DE TEMA

\title{
CONTROVERSIAS EN EL MANEJO QUIRÚURGICO DEL ENDOMETRIOMA OVÁRICO
}

Jaime Saavedra, M.D.*

Recibido: agosto 5/2002 - Revisado: septiembre 2/2002 - Aceptado: noviembre 26/2002

\section{CONTROVERSIES IN THE SURGICAL MANAGEMENT OF OVARIC ENDOMETRIOMAS}

\section{RESUMEN}

La supresión hormonal del endometrioma ovárico mayor de $1 \mathrm{~cm}$ no es efectiva; sin embargo, tal supresión puede mejorar los síntomas en el 40\% a 65\% de los pacientes por un período de 6 a 12 meses. La aspiración de endometrioma ya sea trasvaginal o trasabominal mediante la guía ultrasonográfica o por laparoscopía no es aconsejable como tratamiento quirúrgico de primera línea. Estos procedimientos están seguidos de una alta tasa de recurrencia (28\% a 100\%) dentro de 1 a 9 meses siguientes a la aspiración. La supresión hormonal antes o después de la aspiración no afecta el resultado. La vaporización laparoscópica de las paredes del quiste previa apertura de éste ya sea con láser o electrocauterio es mejor que el tratamiento de aspiración solo. La tasa de mejoría de los síntomas después del procedimiento es de 74\% a 91\%, la tasa de recurrencia es de $8 \%$ a $13 \%$ con una tasa de embarazo del $45 \%$ al $50 \%$.

* Profesor Titular - Universidad Del Valle, Cali - Colombia, Jefe del Servicio de Ginecología, Hospital Universitario, Cali - Colombia, Director del Programa de Entrenamiento en Cirugía Endoscópica Ginecológica, Clínica Los Andes, Cali -Colombia, Presidente de la Sociedad Colombiana de Médicos Endoscopistas, Director General del Centro de Biomedicina Reproductiva - Fecundar, Cali - Secretario Sociedad Iberoamericana de Endoscopia Ginecológica e Imagenología, Siaegi.
El tratamiento laparoscópico del endometrioma ovárico por extirpación de la pared del quiste parece ser el mejor tratamiento quirúrgico. Este procedimiento es técnicamente más difícil que la vaporización de la pared del quiste, pero se asocia con una mejoría prolongada de la sintomatología, baja recurrencia del dolor pélvico, y bajas tasas de reoperación. Las tasas acumulativas de embarazo son más altas que después de vaporización. Los estudios actuales concluyen primero, que la vaporización de la pared interna del endometrioma no daña la función ovárica en términos de los parámetros de FIV y resultados; segundo, que los riesgos teóricos de pérdida de tejido ovárico viable durante la cistectomía existen pero se puede evitar con una técnica microquirúrgica laparoscópica, teniendo el cuidado de preservar la corteza ovárica residual normal; y tercero, los resultados de FIV después de extirpar o destruir los endometriomas en los pacientes con endometriosis son similares cuando se comparan con mujeres con factor tubárico o infertilidad idiopática.

Palabras clave: endometrioma ovárico, tratamiento, tasas de embarazo 


\section{SUMMARY}

The hormonal suppression of ovarian endometriomas larger than $1 \mathrm{~cm}$ in not effective; however, such suppression may improve the symptoms in $40 \%$ to $65 \%$ of the patients for a period of 6 to 12 months. Aspiration of endometriomas either by trasvaginal or transabdominal ultrasound guidance or laparoscopy in no advisable as a first-line surgical treatment. These procedures are followed by a high recurrence rate $(28 \%$ to 100\%) within 1 to 9 months following the aspiration. Hormonal suppression before or after the aspiration does not affect the result. Laparoscopic fenestration and ablation of the endometriótico cyst wall either by laser or electrocautery is a better treatment than aspiration alone. This procedure can be done in a single setting, eliminating the need for another operation. The symptomatic improvement rate after this procedure is $74 \%$ to $91 \%$, the recurrence rate is $8 \%$ to $13 \%$, and the pregnancy rate is $45 \%$ to $50 \%$.

Laparoscopic treatment of ovarian endometrioma by stripping the cyst wall from the remaining ovarian tissue seems to be the best surgical treatment. This procedure is technically more demanding than fenestration, but is associated with prolonged symptomatic improvement, a lower recurrence of pelvic pain, and a lower reoperation rate. The cumulative pregnancy rate is also higher than after fenestration only. The actual studies conclude, first, that vaporization of the internal cyst wall does not impair ovarian function in terms of IVF parameters and outcome; second, that theoretical risks of loss of viable ovarian tissue during cystectomy exist but may be avoided by microsurgical laparoscopic technique, taking care to preserve the normal residual ovarian cortex; and, third, that, after removal or destruction of endometriomas, IVF outcomes are similar in endometriosis patients when compared to women with tubal factor or idiopathic infertility.
Key words: Ovarian endometrioma, treatment, pregnancy rates.

La endometriosis se asocia frecuentemente con infertilidad. Se ha reportado una incidencia del 30 a 70\% de endometriosis en mujeres infértiles, ${ }^{1}$ y una incidencia del 39\% al 59\% en pacientes con dolor pélvico crónico. ${ }^{2-4} \mathrm{El}$ ovario es un sitio común de endometriosis, quizás porque el ovario tiene una superficie irregular, lo cual permite que el tejido endometrial se entierre predisponiendo el desarrollo de quistes endometriósicos o endometriomas.

Algunos reportes de la literatura sugieren que la endometriosis ovárica podría afectar los resultados de fertilización in vitro (FIV). Se ha implicado como causa de ello una pobre respuesta a la estimulación ovárica, una disminución en las tasas de recuperación de ovocitos y en las tasas de fertilización e implantación. ${ }^{5-8}$

El objetivo de esta revisión es hacer claridad sobre el manejo de los endometriomas ováricos con los datos disponibles hasta el momento y evaluar el futuro reproductivo de estas pacientes de acuerdo al procedimiento realizado.

\section{FISIOPATOLOGÍA Y DIAGNÓSTICO}

La fisiopatología del endometrioma ovárico no es clara. Hugshesdon ${ }^{9}$ postuló que el endometrioma se forma por invaginación de la corteza ovárica después de la acumulación de restos de menstruación de implantes endometriales sangrantes sobre la superficie del ovario. Los implantes son cubiertos posteriormente por tejido fibrótico denso.

El endometrioma se manifiesta a menudo por dolor pélvico, infertilidad o una masa anexial.

El diagnóstico se hace usualmente por ultrasonido o al momento de cirugía. 


\section{Tabla 1. Aspiración de endometrioma ovárico transvaginal y transabdominal}

\begin{tabular}{lccc} 
ESTUDIO & ZANETTA et al & GIORIANDINO et al & ABOULGHAR et al \\
\hline \hline Número de pacientes & 18 & 34 & 21 \\
\hline Tipo de estudio & Retrospectivo & Retrospectivo & Prospectivo \\
\hline $\begin{array}{l}\text { Tamaño del } \\
\text { endometrioma }(\mathrm{cm})\end{array}$ & $1,0-9,0(5,5)$ & $3,0-11,0(5,6)$ & $2,6-9,8(4,9)$ \\
\hline Seguimiento (años) & 8 & 1 & 1 \\
\hline Tasa de recurrencia & $100 \%$ & $53 \%$ & $28 \%$ \\
\hline Tipo de drenaje & ATV & ATV y ATA & ATV \\
\hline
\end{tabular}

ATV: Aspiración transvaginal

ATA: Aspiración transabdominal

La visualización a la laparoscopia o laparotomía tiene una sensibilidad de $97 \%$ y una especificidad del $95 \% ;{ }^{10}$ el ultrasonido transvaginal tiene una sensibilidad de $82 \%$ a $89 \%$ y una especificidad del 90\% al 97\% ${ }^{11-14}$, la resonancia magnética (RM) tiene una sensibilidad del $90 \%$ y una especificidad del $98 \%$.

El ultrasonido transvaginal doppler color o pulsado o la determinación de CA-125 no mejora la precisión del diagnóstico por ultrasonido. ${ }^{11}$ El diagnóstico debe ser establecido por estudio histopatológico.

\section{TRATAMIENTO MÉDICO}

El tratamiento médico del endometrioma ovárico con supresión hormonal (progestinas, análogos de la GnRH o danazol) se asocia a una mejoría de la sintomatología en un 40\% a 65\% de las pacientes; sin embargo los síntomas tienden a recurrir a los 6 a 12 meses de haber suspendido el tratamiento. ${ }^{15-16}$ Como es lo esperado, el tratamiento no tiene ningún efecto sobre las adherencias. Además de esto, no es efectivo para endometriomas mayores de $1 \mathrm{~cm} \cdot{ }^{17}$

La terapia médica se ha recomendado como preparación prequirúrgica del endometrioma ovárico. Donnez y cols. ${ }^{18}$ han sugerido que el tratamiento preoperatorio de 3 meses de análogos de GnRH reduce la vascularización y el tamaño del endometrioma, y disminuye el proceso inflamatorio, facilitando la cirugía. En contraste, Mussi y cols. ${ }^{19}$ reportaron que el tratamiento prequirúrgico con análogos de GnRH no ofrece ninguna ventaja en la cirugía o en las tasas de recurrencia. Por tanto, el uso de la terapia médica preoperatoria no parece ser efectiva.

No obstante, ésta puede ser indicada en algunos casos de mujeres con enfermedad extensiva, sintomáticas, como una terapia adicional durante el periodo de espera para la cirugía.

\section{ASPIRACIÓN DEL ENDOMETRIOMA BAJO LA GUÍA DEL ULTRASONIDO}

La aspiración transvaginal se ha recomendado como una alternativa de tratamiento para los endometriomas recurrentes en mujeres con contraindicaciones para la cirugía o anestesia. Los síntomas mejoran usualmente en el primer año después del tratamiento, pero su recurrencia es alta (28\% a 100\%). (Tabla 1)

La presencia de endometriomas ováricos puede afectar los resultados de fertilización in vitro y transferencia de los embriones. Las mujeres en las cuales el contenido del endometrioma fue aspirado primero, el número de folículos aspirados y las 


\begin{tabular}{|c|c|c|c|}
\hline ESTUDIO & MARANA et al & FAYEZ and VOGEL & VERCELLINI et al \\
\hline Número de pacientes & 31 & 44 & 33 \\
\hline Tipo de Estudio & Retrospectivo & Prospectivo & Prospectivo randomizado \\
\hline Tratamiento Concomitante & Ninguno & Danazol & a GnRH \\
\hline Tamaño del Endometrioma $(\mathrm{cm})$ & $3,0-14,7 \quad(5,2)$ & $1,0-9,0$ & $2,0-7,0$ \\
\hline Seguimiento (meses) & 6 & 2 & 6 \\
\hline Tasa de Recurrencia & $84 \%$ & $21 \%$ & $88 \%$ \\
\hline
\end{tabular}

a GnRH: Análogo de la hormona liberadora de gonadotropinas.

tasas de embarazo fueron más altas que en el grupo control. ${ }^{20}$ La presencia de endometriomas ováricos puede hacer técnicamente más difícil la aspiración de los folículos.

Debido a la alta recurrencia, la aspiración del endometrioma ya sea trasvaginal o trasabdominalmente debería realizarse únicamente antes de la fertilización in vitro o cuando la cirugía está contraindicada o no es deseada.

La aspiración de los endometriomas se ha asociado con la formación de adherencias y absceso pélvico. ${ }^{21-22}$

\section{ASPIRACIÓN LAPAROSCÓPICA DEL ENDOMETRIOMA}

La aspiración laparoscópica del endometrioma ha sido realizada por algunos clínicos ${ }^{23,24,25}$ en contraste con la aspiración realizada bajo la guía del ultrasonido, la aspiración bajo control laparoscópico se puede realizar repetidamente, y el contenido del endometrioma derramado, se puede irrigar y remover. Otra de las ventajas incluye la posibilidad de explorar toda la cavidad abdominal. El diagnóstico es mas preciso y se puede establecer el grado de la enfermedad. Dos estudios prospectivos ${ }^{23,25}$ han mostrado que la simple aspiración laparoscópica seguida de supresión médica se encuentra asociada con una tasa de recurrencia del $21 \%$ a los 2 meses de seguimiento y una recurrencia del $88 \%$ a los 6 meses de seguimiento. (Tabla 2).

El número pequeño de pacientes en los estudios así como la variación en el tiempo de seguimiento, hace difícil interpretar los resultados. No obstante es claro que la aspiración laparoscópica del endometrioma ovárico está asociada con una alta tasa de recurrencia tan temprano como a los 2 meses del procedimiento.

\section{MANEJO QUIRÚRGICO DE LOS ENDOMETRIOMAS}

Los endometriomas ováricos requieren cirugía en las mujeres infértiles o en aquellas que presentan sensación de masa o dolor pélvico crónico. Algunos reportes han sugerido recientemente que la cirugía del ovario en caso de endometriomas podría ser deletérea para la corteza ovárica residual normal.

En la literatura, se han descrito dos técnicas quirúrgicas:

1. Vaporización laparoscópica de las paredes internas del quiste después de la apertura de éste.

2. Cistectomía laparoscópica del ovario, lo cual implica quitar las paredes del quiste. 


\begin{tabular}{|c|c|c|c|}
\hline ESTUDIO & HASSON,1990 & DONNEZ et al, 1996 & SUTTON et al, 1997 \\
\hline Período de seguimiento & $1995-1988$ & 1982-1994 & 1983-1993 \\
\hline Número de pacientes & 83 & 607 & 64 \\
\hline Edad (años) & $15-77(32,8)$ & ND & $20-49$ \\
\hline Tipo de vaporización & $\begin{array}{c}\text { Láser de Nd:YAG con } \\
\text { coagulación bipolar }\end{array}$ & Láser de $\mathrm{CO}_{2}$ & Láser de $\mathrm{CO}_{2} / \mathrm{KTP}$ \\
\hline Tamaño de endometriomas (cm) & $2-8$ & $3-15$ & $2-18$ \\
\hline Mejoría síntomas & $32(91)$ & ND & $90(74)$ \\
\hline Tasa de embarazo & ND & 51 & $30(45)$ \\
\hline Tasa de recurrencia & $2(11)$ & $51(8)$ & $(12,5)$ \\
\hline
\end{tabular}

ND: No disponible; Nd:YAG: Neodimio:Yttrium - Silicato de aluminio; CO2/KTP: Dióxido de carbono/Fostato de titanio potásico.

\section{APERTURA Y VAPORIZACIÓN LAPAROSCÓPICA DEL ENDOMETRIOMA}

La apertura se realiza mediante una incisión de 2 cm o más en la pared del quiste, seguida de vaporización del revestimiento interno de las paredes del quiste con láser o electrocauterio.

Donnez y cols. ${ }^{18}$ estudiaron el efecto de análogos de GnRH después de la vaporización laparoscópica y drenaje del endometrioma. El tamaño del endometrioma se redujo aproximadamente en un 50\% después de 12 semanas de tratamiento con el análogo; el endometrioma reaparece rápidamente en pacientes sin tratamiento.

Aunque el tratamiento con análogos de GnRH se asocia con una reducción significativa en el tamaño del endometrioma, todos los pacientes requirieron posteriormente extirpación de la pared del quiste.

Los resultados de la apertura del endometrioma y posterior vaporización de las paredes del quiste se describen en la Tabla 3. La tasa de mejoría de los síntomas reportada es del 74\% al 91\%, la tasa de recurrencia es del $8 \%$ al $9 \%$ y la tasa de embarazo es del 45\% al 50\%. Muchas de las pacientes se embarazaron dentro de los 12 meses siguientes a la cirugía. ${ }^{26}$
La vaporización de las paredes del quiste endometriósico ya sea con láser o electrocauterio, después que el contenido del endometrioma se ha aspirado, parece ser mejor tratamiento que el drenaje solo.

Este tratamiento se puede realizar en una sola ejecución, eliminando la necesidad de otra cirugía. La supresión hormonal ya sea antes o después del procedimiento no es necesaria. ${ }^{19,23,27}$

\section{CISTECTOMÍA \\ LAPAROSCÓPICA DEL ENDOMETRIOMA}

Mettler y Semm ${ }^{28}$ fueron los primeros que realizaron la remoción laparoscópica de la pared del quiste endometriósico. En esta técnica, la escisión se lleva acabo drenando el contenido del endometrioma y luego se retira la pared del quiste del tejido ovárico restante. Los resultados son comparables con los de laparotomía. (Tabla 4) 29-32.

La cirugía laparoscópica tiene las ventajas de menor tiempo quirúrgico, corta hospitalización, baja morbilidad y tiempo de recuperación rápida.

La recurrencia después del procedimiento está entre el 5\% al 12\%. La comparación entre las diferentes modalidades quirúrgicas, escisión con ti- 


\begin{tabular}{lcccccccc} 
& \multicolumn{1}{c}{ Tabla 4. Extirpación de la pared del endometrioma por laparoscopia y laparotomía } \\
\multicolumn{1}{c}{ ESTUDIO } & BATERMAN et al & CATALANO et al & WOOD et al & \multicolumn{2}{c}{ ADAMSON et al } \\
\hline \hline & Laparoscopia & Laparotomía & Laparoscopia & Laparotomía & Laparoscopia & Laparotomía & Laparoscopia & Laparotomía \\
\hline Número de pacientes & 36 & 21 & 83 & 49 & 25 & 27 & 48 & 52 \\
\hline Edad (años) & $33+/-4$ & $34+/-4$ & $18-39$ & $17-38$ & $\mathrm{ND}$ & $\mathrm{ND}$ & $25-46$ & $26-40$ \\
\hline $\begin{array}{l}\text { Tamaño del } \\
\text { endometrioma (cm) }\end{array}$ & $2-9$ & $2-9$ & $3-10$ & $3-16$ & $\mathrm{ND}$ & $\mathrm{ND}$ & $1-8$ & $1-35$ \\
\hline Pacientes con infertilidad & 21 & 15 & 11 & 16 & 25 & 27 & 48 & 52 \\
\hline Mejoría de los síntomas (\%) & 92 & 85 & 66 & 73 & $\mathrm{ND}$ & $\mathrm{ND}$ & $\mathrm{ND}$ & $\mathrm{ND}$ \\
\hline Tasa de recurrencia (\%) & $4(11)$ & $4(19)$ & $4(5)$ & $2(4)$ & $3(12)$ & $2(7)$ & $\mathrm{ND}$ & $\mathrm{ND}$ \\
\hline Tasa de embarazo (\%) & $9(43)$ & $7(47)$ & $5(45)$ & $7(44)$ & $14(56)$ & $12(44)$ & $18(38)$ & $18(35)$ \\
\hline $\begin{array}{l}\text { Tiempo de } \\
\text { seguimiento (años) }\end{array}$ & 1 & 1 & 3 & 3 & 1 & & 1 & 3 \\
\hline \hline
\end{tabular}

ND: No disponible.

jeras y electrocauterio o láser produce iguales resultados. (Tabla 5 y 6).

El método de quitar la pared del quiste permite una completa remoción del endometrioma y se asocia con una baja tasa de recurrencia a los 5 años. ${ }^{33}$

\section{VAPORIZACIÓN DE \\ LA PARED DEL QUISTE ENDOMETRIÓSICO VERSUS EXTIRPACIÓN DE LA PARED DEL QUISTE}

Fayez y Vogel $^{23}$ evaluaron los diferentes tratamientos laparoscópicos del endometrioma ovárico; el seguimiento fue corto y todos los pacientes recibieron danazol en el postoperatorio. Las tasas de embarazos en los casos de extirpación de la pared del quiste fueron similares a la de los casos de vaporización de la pared interna del quiste. ${ }^{34}$ En un estudio prospectivo y aleatorizado realizado por Beretta y cols. ${ }^{35}$ se demostró que la tasa de recurrencia, dispareunia y dolor pélvico fue significativamente más baja en los pacientes a los cuales se les realizó extirpación de la pared del quiste, que a los que se les practicó vaporización. El tiempo de intérvalo de recurrencia del dolor fue mayor en los casos de extirpación (19 meses) comparado con el grupo de vaporización $(9,5$ meses). La tasa acumulativa de embarazo a los 24 meses fue de 66,7\% en el grupo de extirpación versus $23,5 \%$ en el grupo al que se le realizó vaporización, la tasa de recurrencia del endometrioma fue $6,2 \%$ en el grupo de extirpación versus $18,8 \%$ en grupo de vaporización.

La tasa de reoperación después de extirpación laparoscópica del endometrioma ovárico fue significativamente más baja que después de vaporización laparoscópica.

\section{RESULTADOS DE FERTILIZACIÓN IN VITRO DESPUÉS DE LA CIRUGÍA DEL ENDOMETRIOMA}

Wyns y cols. ${ }^{36}$ evaluaron si la cirugía del endometrioma (cistectomía o vaporización de la pared del quiste) pudiese perjudicar los resultados de fertilización in vitro (FIV). Ellos analizaron la respuesta a la estimulación con gonadotropinas para FIV en mujeres que habían tenido cirugía laparoscópica para endometriomas pero que no habían llegado a embarazarse al año de cirugía.

El estudio comprometió a 447 mujeres que fueron a FIV, de éstas 119 con endometriosis probada, quienes no se habían embarazado después 


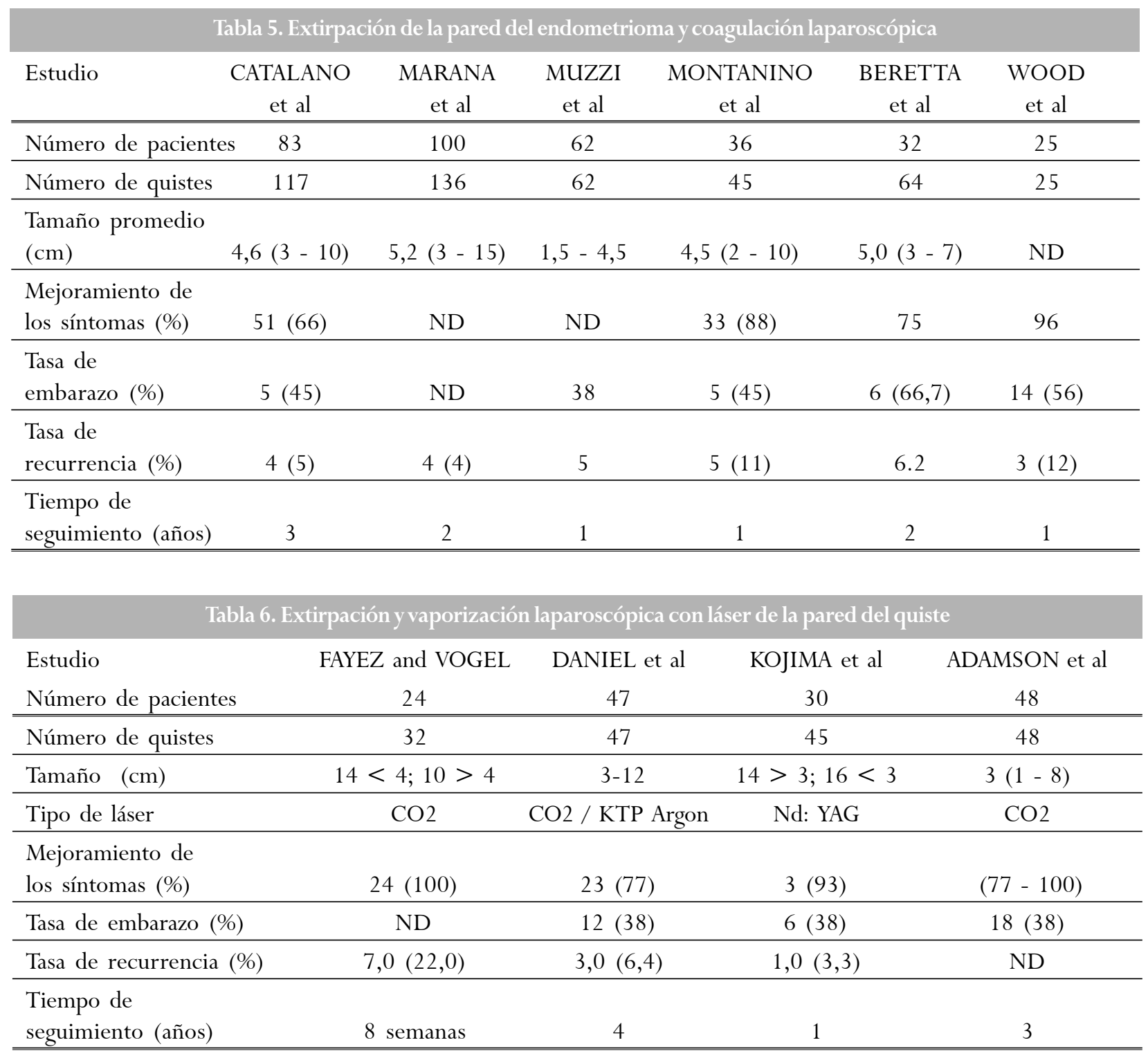

de una cirugía laparoscópica conservadora de endometriosis.

El conjunto de pacientes con endometriosis se dividió en tres grupos: grupo Ia, 42 pacientes con lesiones peritoneales tratadas por laparoscopia con destrucción de los implantes (vaporización o coagulación), grupo $\mathbf{I b}, 57$ pacientes con endometriomas ováricos tratados por vaporización de la pared del quiste, un grupo Ic, 20 mujeres con endometriomas tratados con extirpación de la pared del quiste.
El grupo control (grupo II), incluyó 328 pacientes que fueron a FIV durante el mismo periodo, éste fue dividido en dos subgrupos: grupo IIa, 193 mujeres con factor tubárico, grupo IIb, 135 pacientes con infertilidad hidropática.

Los parámetros de estimulación se muestran en la Tabla 7. No hubo diferencia entre los subgrupos del estudio, en cuanto a número de ampollas de gonadotropinas, número de folículos mayores de $15 \mathrm{~mm}$ de diámetro, número de oocitos maduros, $\mathrm{y}$ concentraciones de $\mathrm{E}_{2}$. 


\section{Tabla 7.Parámetros de estimulación ovárica en pacientes con}

endometriomas según procedimiento quirúrgico antes de FIV

\begin{tabular}{|c|c|c|c|c|c|}
\hline & \multicolumn{3}{|c|}{ Endometriosis } & \multicolumn{2}{|c|}{ No endometriosis } \\
\hline & \multicolumn{3}{|c|}{ Grupo 1} & \multicolumn{2}{|c|}{ Grupo 2} \\
\hline & Peritoneal & $\begin{array}{l}\text { Quiste ovárico } \\
\text { vaporización } \\
\text { de la pared }\end{array}$ & $\begin{array}{c}\text { Cistectomía } \\
\text { endometrioma }\end{array}$ & Tubárico & Idiopático \\
\hline & (Grupo 1a) & (Grupo 1b) & (Grupo 1c) & (Grupo 2a) & (Grupo 2b) \\
\hline Número & 42 & 57 & 20 & 193 & 135 \\
\hline Ciclos & 71 & 125 & 38 & 422 & 275 \\
\hline Ampollas & $36,52+/-13,97$ & $41,34+/-18,93$ & $40,61+/-16,20$ & $44,17+/-19,76$ & $39,74+/-19,98$ \\
\hline Folículos & $14,67+/-8,04$ & $15,90+/-6,62$ & $14,44+/-6,41$ & $12,96+/-6,82$ & $15,57+/-9,70$ \\
\hline Oocitos maduros & $9,66+/-5,92$ & $10,90+/-4,91$ & $9,29+/-4,52$ & $8,55+/-6,99$ & $9,44+/-6,13$ \\
\hline Día del hCG & $12,46+/-1,54$ & $12,97+/-2,30$ & $13,03+/-2,31$ & $13,08+/-2,58$ & $13,18+/-2,70$ \\
\hline
\end{tabular}

\section{Tabla 8. Resultados de FIV en pacientes con endometrioma después de cirugía}

Endometriosis

No endometriosis

\begin{tabular}{lccccc}
\hline & & Grupo 1 & & Go endometriosis \\
\hline \hline & Peritoneal & $\begin{array}{c}\text { Quiste ovárico } \\
\text { vaporización } \\
\text { de la pared }\end{array}$ & $\begin{array}{c}\text { Cistectomía } \\
\text { endometrioma }\end{array}$ & Tubárico & Idiopático \\
& $($ Grupo 1a) & (Grupo 1b) & (Grupo 1c) & (Grupo 2a) & (Grupo 2b) \\
\hline Tasa de fertilización & $6,81 \%$ & $57,43 \%$ & $64,59 \%$ & $62,48 \%$ & $57,99 \%$ \\
\hline Embriones/Oocitos & $45,19 \%$ & $41,31 \%$ & $41,08 \%$ & $43,50 \%$ & $58,37 \%$ \\
\hline maduros & $4,19+/-4,69$ & $4,26+/-3,33$ & $3,82+/-3,09$ & $3,72+/-3,95$ & $3,82+/-3,98$ \\
\hline Embriones / Ciclo & $2,26+/-1,10$ & $2,57+/-0,92$ & $2,63+/-1,63$ & $2,35+/-1,08$ & $2,23+/-1,13$ \\
\hline Transferidos & $17,72 \%$ & $14,95 \%$ & $25,93 \%$ & $13,94 \%$ & $18,05 \%$ \\
\hline Tasa de implantación & $32,39 \%$ & $36,80 \%$ & $50 \%$ & $27,49 \%$ & $30,18 \%$ \\
\hline Tasa de embarazos & & & & & \\
\hline \hline
\end{tabular}

En la Tabla 8 se muestra el número de embriones/ciclo, el número de embriones trasferidos/ciclo, tasa de fertilización, e implantación y embarazos en curso. No hubo diferencia significativa entre los diferentes subgrupos en los parámetros estudiados.

Con el propósito de establecer si el tamaño del endometrioma removido o vaporizado pudiese influenciar el resultado de FIV en la Tabla 9 se analizaron varios parámetros de acuerdo con el tamaño del endometrioma, $(<3 \mathrm{~cm}$ versus $>3$ cm), no se observó ninguna diferencia entre los grupos Ib y Ic.

Se analizaron pacientes con endometriomas unilaterales y ovario contralateral normal. El número de folículos $>15 \mathrm{~mm}$ en tamaño, el número total de folículos, y el número de oocitos fueron similares en el ovario tratado y en ovario contralateral normal. (Tabla 10). 


\begin{tabular}{|c|c|c|c|c|}
\hline & \multicolumn{2}{|c|}{ Quiste ovárico vaporizado } & \multicolumn{2}{|c|}{ Cistectomía del endometrioma } \\
\hline & \multicolumn{2}{|c|}{ Grupo $1 \mathrm{~b}$} & \multicolumn{2}{|c|}{ Grupo $1 \mathrm{c}$} \\
\hline & $<3 \mathrm{~cm}$ & $>3 \mathrm{~cm}$ & $<3 \mathrm{~cm}$ & $>3 \mathrm{~cm}$ \\
\hline Edad & $31,56+/-3,81$ & $31,81+/-3,50$ & $33,14+/-3,00$ & $31,17+/-4,46$ \\
\hline Tamaño promedio & $1,48+/-0,58$ & $5,51+/-2,48$ & $1,46+/-0,67$ & $5,96+/-2,10$ \\
\hline Número total de folículos & $14,21+/-6,72$ & $17,76+/-6,62$ & $13,43+/-6,70$ & $15,92+/-7,22$ \\
\hline Número folículos $>15 \mathrm{~mm}$ & $10,96+/-5,34$ & $13,62+/-6,17$ & $10,00+/-4,45$ & $12,62+/-6,48$ \\
\hline
\end{tabular}

\begin{tabular}{|c|c|c|c|c|}
\hline & \multicolumn{2}{|c|}{ Vaporización de la pared del quiste } & \multicolumn{2}{|c|}{ Cistectomía del endometrioma } \\
\hline & \multicolumn{2}{|c|}{ Grupo 1b } & \multicolumn{2}{|c|}{ Grupo 1c } \\
\hline & \multicolumn{2}{|c|}{$(n=57$ ciclos $)$} & \multicolumn{2}{|c|}{$(\mathrm{n}=20$ ciclos $)$} \\
\hline & Ovario normal & Endometrioma & Ovario normal & Endometrioma \\
\hline Edad & $31,53+/-3,61$ & $31,53+/-3,61$ & $32,14+/-3,47$ & $32,14+/-3,47$ \\
\hline Tamaño promedio del quiste & - & $2,66+/-1,83$ & - & $3,69+/-2,71$ \\
\hline Número total de folículos & $8,29+/-4,17$ & $6,82+/-3,66$ & $6,57+/-5,80$ & $7+/-4,90$ \\
\hline Número folículos $>15 \mathrm{~mm}$ & $6,50+/-3,91$ & $4,94+/-3,16$ & $4,07+/-4,98$ & $5,28+/-3,93$ \\
\hline Número de oocitos & $5,31+/-3,02$ & $4,39+/-2,91$ & $3,64+/-3,24$ & $4,51+/-3,37$ \\
\hline
\end{tabular}

\section{DISCUSIÓN}

Podemos decir que el manejo de los quistes endometriósicos es aún objeto de debate y controversia. Algunos autores han sugerido que la cistectomía podría provocar pérdida del tejido ovárico por remoción del estroma ovárico con oocitos junto con la cápsula o por daño térmico provocado por la coagulación. ${ }^{24,34}$

Hemming y cols. ${ }^{34}$ correlacionan la rápida concepción y unas mejores tasas de embarazo en pacientes tratadas por coagulación de la pared del quiste comparado a cistectomía con la menor perdida considerable de tejido ovárico.

Donnez y cols. sugirieron que el retirar la pared del endometrioma puede resultar en pérdida de corteza ovárica viable durante la cirugía. ${ }^{26}$ En un estudio histológico este autor demostró ausencia de plano de clivaje, lo cual hace difícil quitar la pared del quiste, dando origen a la posibilidad de sangrado en el hilio, requiriendo una coagulación excesiva ${ }^{24,37}$. De acuerdo con la teoría de la metaplasia de invaginación del mesotelio, los oocitos se encuentran densamente rodeando la cápsula del endometrioma, y por tanto hay el riesgo teórico de llevarse los oocitos junto con la cápsula del endometrioma ${ }^{38,39}$ En estudios histológicos del endometrioma extirpado, se demostró que los oocitos se encuentran en el tejido extirpado. ${ }^{35,36}$

Loh y cols. ${ }^{40}$ demostraron que los ovarios postcistectomía presentan una baja respuesta folicular en ciclo natural y al estímulo con clomifeno, pero producen un número comparable de folículos al ovario normal cuando se estimulan con gonadotropinas.

Los resultados de Wyns y cols. ${ }^{36}$ muestran que la estimulación ovárica tanto en cistectomía como en vaporización de la pared del endometrioma se 
conserva produciendo un número comparable de folículos al tejido ovárico normal cuando se estimula con gonadotropinas.

Esto prueba que la vaporización y la cistectomia, cuando se realizan cuidadosamente por manos expertas, no afectan la respuesta del ovario en cuanto a producir oocitos maduros, proporcionando una evidencia indirecta de la persistencia de corteza ovárica viable, similar al tejido ovárico normal; incluso si el tamaño $(<$ ó $>$ de $3 \mathrm{~cm}$ ) del endometrioma se toma en cuenta, no se observa ninguna diferencia.

Se ha demostrado claramente que la tasa de fertilización no se afecta en las mujeres con endometriosis y que la cirugía del endometrioma no interfiere con la tasa de fertilización. ${ }^{36}$ Por tanto, si la tasa de fertilización se encuentra alterada en mujeres con endometriomas como lo reclaman algunos autores, ésta puede ser normalizada por la cirugía. $7,8,41,42$

\section{CONCLUSIONES}

1. La supresión hormonal de endometriomas mayores de $1 \mathrm{~cm}$ como tratamiento no es efectiva, pero mejora los síntomas.

2. La aspiración del endometrioma ya sea transabdominal, transvaginal o por laparoscopia no debe ser tratamiento de primera línea, ya que la tasa de recurrencia es muy alta.

3. La vaporización de la pared del quiste da mejores resultados que la aspiración, presentando baja tasa de recurrencia de endometrioma.

4. La cistectomía laparoscópica es el mejor tratamiento, presenta tasas de embarazo más altas que la vaporización de la pared del quiste.

5. La vaporización de la pared del endometrioma no daña la función ovárica para FIV.

6. Los riesgos teóricos de la pérdida de tejido ovárico viable durante la cistectomía se pueden evitar con técnica microquirúrgica laparoscópica.
7. Los resultados en FIV después de tratar quirúrgicamente a los endometriomas son similares a la de los pacientes con factor tubárico o infertilidad idiopática.

\section{BIBLIOGRAFÍA}

1. Nisolle M, Donnez J. Peritoneal endometriosis, ovarian endometriosis and adenomyotic nodules of the rectovaginal septum are three distinct entities. Fertil Steril 1997; 68:585-596A.

2. Buttran VC Jr. Conservative surgery for endometriosis in the fertile female: a study of 206 patients with implications for both medical and surgical therapy. Fertil Steril 1979; 42:117-123.

3. Fedele L, Parazzini F, Bianchi S, et al. Stage and location of pelvic endometriosis and pain. Fertil Steril 1990; 53:166-170.

4. Mahmood TA, Templeton A. Prevalence and genesis of endometriosis. Hum Reprod 1991; 6:544-549.

5. Arici A, Oral E, Bukulmez O, et al. The effect of endometriosis on implantation: results from the Yale University in vitro fertilization and embryo transfer program. Fertil Steril 1996; 65:603-607.

6. P, Vendola N, Bocciolone L, et al. Reliability of the visual diagnosis of ovarian; Cahill DJ, Wardle PG, Maile LA, et al. Ovarian dysfunction in endometriosis - associated and unexplained infertility. J Asst Reprod Genet 1997; 14:554-557.

7. Yanushpolsky EH, Best CL, Jacson KV, et al. Effects of endometriomas on oocyte quality, embryo quality, and pregnancy rates in vitro fertilization cycles: a prospective case - controlled study. J Asst Reprod Genet 1998; 15:27-31.

8. Pal L, Shifren JL, Isaacson KB, et al. Impact of varying stages of endometriosis on the outcome of in vitro fertilization - embryo transfer. J Assist Reprod Genet 1998; 15:27-31.

9. Hughesdon PE. The structure of endometriotic cysts of the ovary. J Obstet Gynaecol Br Emp 1957; 44:481487.

10. Vercellini P, Vendola N, Bocciolone L, et al. Reliability of visual diagnosis of ovarian endometriosis. Fertil Steril 1991; 56:1198-1200.

11. Alcazar JL, Laparte C, Jurado M, et al. The role of transvaginal sonography combined with color velocity 
imaging and pulsed Doppler in the diagnosis of endometriomas. Fertil Steril 1997; 67:487-491.

12.Guerriero S, Mais V, Ajossa S, et al. Trasvaginal ultrasonography combined with CA 125 plasma level in the diagnosis of endometriomas. Fertil Steril 1996; 65:293-298.

13. Mais V, Guerriero S, Ajossa S, et al. The efficiency of trasvaginal ultrasonography in the diagnosis of endometriomas. Fertil Steril 1993; 60:776-780.

14. Volpi E, Maher P, Hill D. Diagnosis and surgical management of endometriomas. Aust NZ J Obstet Gynaecol 1992; 32:161-163.

15. Schenken RS. Gonadotropin releasing hormone analogs in the treatment of endometriomas. Am J Obstet Gynecol 1990; 162:579-581.

16.16. Shaw RT. Treatment of endometriosis. Lancet 1992; 340:1267-1271.

17. Shaw RW. The role of GnRH analogues in the treatment of endometriosis. Br J Obstet Gynaecol 1992; 99:9-12.

18.Donnez J, Nisolle M, Gillerot S, et al. Ovarian endometrial cysts: the role of gonadotropin releasing hormone agonists and/or drainage. Fertil Steril 1994; 62:63-66.

19. Muzii L, Marana R, Caruana P, et al. The impact of preoperative gonadotropin releasing hormone agonist treatment on laparoscopic excision of ovarian endometriotic cysts. Fertil Steril 1996; 65:1235-1237.

20.Dicker D, Goldman JA, Feldeberg D, et al. Transvaginal ultrasonic needle - guided aspiration of endometriotic cysts before ovulation induction for in vitro fertilization. J Vitro Fertil Embryo Transfer 1991; 8:286-289.

21. Muzii L, Marana R, Caruana P, et al. Laparoscopic findings after transvaginal ultrasound-guided aspiration of ovarian endometriomas. Hum Reprod 1995; 10:2902-2903.

22. Padilla SI. Ovarian abscess following puncture of an endometrioma during ultrasound - guided retrieval. Hum Reprod 1993; 8:1282-1283.

23. Fayez JA, Vogel MS. Comparison of different treatment methods of endometriosis by laparoscopy. Obstet Gynecol 1991; 78:660-665.

24. Marana R, Caruane P, Muzii L, et al. Operative laparoscopy for ovarian cysts: excision versus aspiration. J Reprod Med 1996; 41:435-438.
25. Vercellini P, Vendola N, Bocciolone L, et al. Laparoscopic aspiration of ovarian endometriomas: effect with postoperative gonadotropin releasing hormone agonist treatment. J Reprod Med 1992; 37:577-580.

26.Donnez J, Nisolle M, Gillet N, et al. Large endometrioma. Hum Reprod 1996; 11:641-646.

27. Sutton CJ, Ewen SP, Jacobs SA, et al. Laser laparoscopic surgery in the treatment of ovarian endometrioma. J Am Assoc Gynecol Laparoscop 1997; 4:319-323.

28. Mettler L, Semm K. Three step medical and surgical treatment of endometriosis. Isr J Med Sci 1983; 152:24.

29.Adamson GD, Subak LL, Pasta DJ. Comparison of $\mathrm{CO}_{2}$ lasers laparoscopy with laparotomy for treatment of endometriomata. Fertil Steril 1992, 57:965-973.

30. Bateman BG, Kolp LA, Mills S. Endoscopic versus laparotomy management of endometriomas. Fertil Steril 1994; 62:690-695.

31. Catalano GF, Marana R, Caruana P, et al. Laparosocopy versus microsurgery by laparotomy for excision of ovarian cysts in patients with moderate or severe endometriosis. J Am Assoc Gynecol Laparoscop 1996; 3:267-270.

32. Wood C, Maher P, Hill D. Diagnosis and surgical management of endometriomas. Aust NZ J Obstet Gynaecol 1992; 32:161-163.

33. Redwine DB. Conservative laparoscopic excision of endometriosis by sharp dissection: life table analysis of reopertion and persistent or recurrent disease. Fertil Steril 1991; 56:628-634.

34.Hemmings R, Bissonnette F, Bouzayen R. Results of laparoscopic treatments of ovarian endometriomas: laparoscopic ovarian fenestration and coagulation. Fertil Steril 1998; 70:527-529.

35.Beretta P, Franchi M, Ghezzi F, et al. Randomized clinical trial of two laparoscopic treatments of endometriomas: cystectomy versus drainage and coagulation. Fertil Steril 1998; 70:1176-1180.

36. Wyns C, Pirard C, Nisolle M, et al. In vitro fertilization outcome after endometrioma surgery. En: Donnez J, Nisolle M, Eds. An atlas of operative laparoscopiy and histeroscopy. UK: Parthenon Publishing Group 2001:77-81. 
37. Donnez J, Nisolle M, Anaf V, et al. Endoscopic management of peritoneal and ovarian endometriosis En: Donnez J, Nisolle M, eds. An Atlas of Laser Operative Laparoscopic and Histeroscopy. Camforth, UK: Parthenon Publishing, 1994: p. 53-74

38. Nisolle M, Donnez J. Peritoneal endometriosis, ovarian endometriosis and adenomyotic nodules of the rectovaginal septum are three distinct entities. Fertil Steril 1997; 68:585-596

39. Nisolle M, Casanas-Roux F, Donnez J. Large ovarian endometriomas: new perspectives in histiogenesis. Ref Gyn/Obst 1996; 4:381-388
40.Loh FH, Tan AT, Kumar J et al. Ovarian response after laparoscopic ovarian cystectomy for endometriotic cysts in 132 monitored cycles. Fertil Steril 1999; 72:316-321

41. Wardle PC, Mitchell JD, McLaughlin EA, et al. Endometriosis and ovulatory disorder: reduced fertilization in vitro compared with tubal and unexplained infertility. Lancet 1985; 2:236-239

42. Simon C, Gutierrez A, Vidal A, et al. Outcome of patients with endometriosis in assisted reproduction: results from in vitro fertilization and oocyte donation. Hum Reprod 1994; 9:725-729. 